\title{
Rhesus haemolytic disease
}

\author{
Martin J Whittle
}

Until the late 1960s when anti-D prophylaxis was introduced, stillbirths and neonatal deaths from rhesus isoimmune disease were significant contributors to the perinatal mortality rate, whereas most recent perinatal surveys report virtually no losses. Explanations for this include the crucial role of prophylaxis and the limitation of family size, the problem becoming worse with successive pregnancies.

In part, however, the reduction is artifactual, because some losses occur before 28 weeks and are therefore registered as abortions and not as perinatal deaths.

The reduction in the overall incidence of rhesus disease has meant that experience among obstetricians, paediatricians, general practitioners, and midwives is generally low necessitating the development of expertise concentrated in large regional centres from which management advice can be readily available. It should be remembered, however, that prevention is the most effective method of 'managing' rhesus disease, so it is vital to ensure that anti-D is administered at the correct time and in the correct dose after any potentially sensitising event.

Events that may cause sensitisation in rhesus negative women if rhesus positive blood leaks into their circulation

Abortion:

Threatened

Spontaneous

Spontaneous
Induced

Ectopic pregnancy

Procedure related: Chorionic villus sampling Amniocentesis

Fetal blood sampling Intrauterine surgery

Obstetric:

Antepartum haemorrhage External cephalic version Delivery

The Queen Mother's Hospital, Glasgow

Correspondence to: Professor M J Whittle, Department of Fetal Medicine Birmingham Maternity Hospital, Edgbaston,

Birmingham B15 2TG.

\section{Pathophysiology}

Classically rhesus disease occurs because maternal anti-D antibodies cross the placenta and cause haemolysis of rhesus positive fetal red cells. Sensitisation occurs in rhesus negative women if rhesus positive fetal blood leaks into their circulation either spontaneously or by one of the events shown in the table. Most commonly leakage occurs when the placenta separates at delivery, and hence the rationale for anti-D administration (in a dose of at least $500 \mathrm{IU}$ ) within 72 hours of this event. Antibodies may develop during the first pregnancy probably as a result of spontaneous leakage, and the prophylactic use of anti-D during pregnancy (usually at 28 and 34 weeks) to neutralise fetal cells, has been attempted.

Though anti-D is the most common antibody, others also produce disease including $\mathrm{Rh}$ C, D, E, c, and e, Kell, Kidd, and Duffy antibodies. All are IgG in configuration and capable of crossing the placental barrier. Regardless of type their haemolytic ability relates to their avidity, absolute concentrations being less important. This uncertainty about antibody activity means that great care must be taken when planning management. Relatively low concentrations of antibodies are sometimes capable of producing severe disease.
Steadily increasing fetal anaemia is almost certainly the result of haemolysis although there is a suggestion that anti-Kell, for example, may also suppress erythropoiesis. Extramedullary erythropoiesis starts in response to the anaemia, one effect of which is fetal hepatosplenomegaly. The liver becomes totally dedicated to the production of red cells and other activities are obtunded. Thus reduced albumin manufacture leads to hypoalbuminaemia, a fall in plasma oncotic pressure, and the development of hydrops. Further deterioration progresses to metabolic acidosis, high output cardiac failure, and death.

The timing of these events in pregnancy is to some extent unpredictable. The fetus is rarely affected before 16 weeks, probably because the antibodies do not cross the placenta until then. Thereafter timing depends on antibody type, quantity, and avidity together with the fetus's ability to respond. The point at which the changes become irreversible is also uncertain, though recent experience with a fetus whose first packed cell volume was only 0.04 suggests that cardiac damage may occur at about this point. The fetus survived several intrauterine transfusions and the hydrops resolved, but the baby died of unresponsive heart failure about eight days after delivery.

\section{Assessment}

About $15 \%$ of the population of the western world are rhesus negative, and if anti- $D$ antibodies are not found at the time of booking, further checks should be made throughout pregnancy; the minimum recommendations are at 28 weeks, 34 weeks, and at the time of delivery. If antibodies are found at any time the mother should be recalled and the test repeated, preferably within two weeks. Non-rhesus antibodies will be screened for at the booking visit, and perhaps once more, later in pregnancy, and because they can act atypically their presence should be brought to the attention of the regional centre.

\section{SEROLOGY}

Titres of anti-D antibody below $4 \mathrm{IU} / \mathrm{ml}$ or, when the antibodies are only semiquantified, at or below 1 in 16 are very unlikely to produce serious fetal disease; in general this applies to other antibody systems too.

Once antibodies are present the frequency of measurement depends on their absolute titre and the past history. For example a titre below 
$4 \mathrm{IU} / \mathrm{ml}$ requires measurements every two weeks but above this titre weekly assessments are sometimes desirable.

A titre above the critical $4 \mathrm{IU} / \mathrm{ml}$ (or 1 in 16) will indicate the need for further evaluation and once a value of $10 \mathrm{IU} / \mathrm{ml}$ has been reached advice from a referral centre should be sought. Below $5 \mathrm{IU} / \mathrm{ml}$ only $2 \%$ of babies have mild or moderate anaemia whereas at titres between 20 and $100 \mathrm{IU} / \mathrm{ml}, 10 \%$ may have severe anaemia (defined as a haemoglobin concentration of less than $70 \mathrm{~g} / \mathrm{l})$. When the antibody content exceeds $100 \mathrm{IU} / \mathrm{ml}$, however, about $75 \%$ will have severe anaemia, and virtually all the remainder will have mild to moderate disease.

\section{OBSTETRIC HISTORY}

Traditionally the severity of rhesus disease has been judged from events in the obstetric history, the assumption being made that the disease becomes worse with each pregnancy. In general this is true but mild disease in the last pregnancy-for example, requiring only phototherapy at term-does not insure against the need for an intrauterine transfusion during the next pregnancy. The birth of a baby requiring exchange transfusion, one needing preterm delivery for suspected anaemia, and the miscarriage or stillbirth of a hydropic fetus, is particularly ominous. Information about earlier pregnancies should always be obtained and the father's blood genotype determined: it is important not to be coy about ensuring that the right partner is identified. The value of the history in planning management has remained controversial, but if the last baby was severely affected and the partner is homozygous for the $D$ antigen it virtually guarantees that the next baby will be at least as badly affected and probably more so. An adage about the management of rhesus disease states that the easiest pregnancy to deal with was the last one.

\section{AMNIOCENTESIS}

The use of amniotic fluid to evaluate rhesus disease was described in the early 1960s and modified by several authorities, each of whom developed a unique protocol and different nomenclature; all of this has led to confusion. Liley, for example, who many regard as the father of fetal medicine, divised zones (upper, mid, lower) that represented risk categories based on the concentration of bilirubin in the amniotic fluid. Robertson retitled these so that the upper zone, in which the risk was highest, became zone 1 and the lower zone, zone 3, was the least affected. Freda in the United States, however, numbered the chart the other way round, designating the upper zone as zone 3 . Another chart, described by Whitfield, superimposed the action line that demarcated Liley's middle and upper zones.

In spite of these difficulties the constitution of amniotic fluid has remained fundamental in the assessment of rhesus disease. In principle, the breakdown products from the haemolysed fetal red cells appear in the amniotic fluid as bilirubin, high concentrations indicating a serious condition. Bilirubin is quantified, usually by spectroscopic analysis, the maximum absorption for bilirubin being at a wavelength of $450 \mathrm{~nm}$. The optical density difference is plotted on the Liley chart. Liley quickly realised that single measurements were of little use and that at least two measurements, preferably 10 to 14 days apart, were required.

Amniocentesis is indicated when anti-D exceeds $4 \mathrm{IU} / \mathrm{ml}$, or a titre of 1 in 16 for other antibody systems. The timing of the first amniocentesis is vital and a rule of thumb is that it should be done 10 weeks before a rhesus related event occurred during the last pregnancy. For example, if the previous baby had to be delivered at 36 weeks' gestation because of a perceived rhesus problem then the amniocentesis in the next pregnancy should be at 26 weeks.

The current value of amniocentesis is debatable mainly because nowadays the more severe disease often becomes manifest between 20 and 24 weeks, when the Liley zones are invalidthey do not start until 27 weeks. Amniotic fluid analysis can give spurious reassurance in early pregnancy but the enormous experience that exists in its use in the evaluation of rhesus disease should not be disregarded and the technique still has a role in the management of the pregnancy at 27 weeks and beyond when the baby is not hydropic.

The use of amniotic fluid has several advantages. Firstly, it gives a better idea of the progress of the disease than does fetal blood sampling. Amniocentesis is a relatively easy procedure, which is probably less likely to stimulate the disease because it is nearly always possible to avoid the placenta. It can be done at most hospitals, although it is often wise to send the sample to the regional laboratory for analysis and interpretation.

\section{ULTRASOUND}

Modern ultrasound equipment allows the detection of early ascites so that with weekly or even twice weekly scans it is possible to delay any intervention, which will certainly be safer done later in the pregnancy; in addition fewer procedures will be required the later in pregnancy they are started. This non-invasive approach needs to be cautious, however, because the reliability of ultrasound in the detection of the early changes of ascites is uncertain and the required frequency of observations is unknown.

The biophysical profile can be used to monitor the fetal condition and evidence that the fetus is moving, breathing, and has good limb tone is welcome. In our experience, however, the profile does not seem to be sufficiently sensitive to detect subtle deteriorations in the fetal condition. The maternal perception of activity is often better, most mothers remarking that their baby moves less before a transfusion and more vigorously afterwards.

The value of Doppler waveform analysis from the fetal aorta and other vessels remains to be confirmed, and claims that a correlation exists 
between the readings and the fetal anaemia are interesting but not clinically useful.

\section{FETAL BLOOD SAMPLING}

The role of blood sampling in the evaluation of rhesus disease is still controversial, although there can be little doubt about its value in severe cases. The initial sample provides precise information about the fetal haematological state and blood type, the latter being important if the father is a heterozygote; if the baby is rhesus negative no further invasive procedures are required.

Although access to the fetal circulation is a major advance there are several important problems. Firstly, though the presence of anaemia indicates the need for transfusion, the course of action is less clear when the haematological state is normal. Arbitrarily the blood is resampled in one to two weeks but even this second sample may give little impression about what to do next. This is in contrast to the results from amniotic fluid, which will usually give a trend. It is theoretically possible for the fetus to maintain its haemoglobin concentration while showing evidence of haemolysis by a rising bilirubin concentration in amniotic fluid. Which test is most appropriate probably depends on the severity of the disease, fetal blood sampling being more valuable in the severest forms. The difficulty is in predicting the severity of each individual case.

\section{Treatment}

The decision to start treatment may be based on ultrasound evidence of ascites, a rising amniotic fluid bilirubin content, or haematological evidence of fetal anaemia, and (as discussed above) the method will depend on the severity of the disease.

The need to treat will depend to some extent on the gestational age and the presence of ascites. Clearly if amniotic fluid is showing a rising level crossing into the upper zone after 36 weeks then, in the absence of hydrops, delivery is undoubtedly the best policy. The development of ascites, even at this late stage is, I believe, an indication to carry out an intrauterine transfusion, because a fetus is more haemodynamically stable than a neonate and can more easily tolerate the transfusion. Once the fetus has been transfused it can be left in utero for a week to $\mathbf{1 0}$ days to stabilise and then be delivered. Certainly the delivery of a hydropic baby with severe anaemia and stiff lungs who requires immediate transfusion is a daunting paediatric problem, which is best avoided.

Intrauterine treatment is most commonly given by intraperitoneal and intravascular routes.

\section{INTRAPERITONEAL TRANSFUSION}

Liley was probably the first to realise that the intraperitoneal route could be used for blood transfusion and it became the paradigm for the treatment of severe rhesus disease.
A needle (usually 16 gauge) was inserted into the fetal peritoneal cavity under radiographic control. Once it was in place, a fine catheter was threaded down the needle into the cavity and the needle removed. The transfusion of concentrated cells was started at the rate of $1 \mathrm{ml} /$ hour, the volume of blood given being calculated arbitrarily from gestational age (gestational age minus 20 multiplied by 10); thus at 32 weeks the required transfusion would be $120 \mathrm{ml}$. The survival rate from this procedure was good providing the fetus was neither hydropic nor too premature. The latter factor arose because of the technical problems associated with placing a needle under radiographic control into a mobile fetus of, say, 24 weeks' gestation. These problems have been largely overcome by using ultrasound guidance, which makes the intraperitoneal route more acceptable for fetuses at earlier gestational ages. Indeed there are advantages in combining intraperitoneal and intravascular transfusions. Unfortunately the intraperitoneal route is rarely, if ever, successful when the fetus has hydrops, probably because the lymphatics through which the blood is transported are waterlogged; this may also occur in simple ascites. The presence of ascites therefore makes intraperitoneal transfusion ineffective, and the intravascular approach has proved lifesaving in these seriously affected cases.

INTRAVASCULAR TRANSFUSION

The development of fetoscopy as a means of guiding the puncture of the funic vessels led to the first effective intravascular transfusions. Subsequently it became apparent that the same procedure could be done under ultrasound control, and since the mid 1980s this has been the method of choice.

Although the technical problems of intrauterine transfusion have been largely overcome a number of issues remain including the timing and frequency of transfusion, the volume of the transfusion and the rate at which it is given, the optimal route and the timing of delivery.

I have already discussed the timing of the first transfusion, and the calculation often involves a number of factors whose inter-relations are complex. Obviously the earlier the transfusion, the more hazardous the procedure even in the most experienced hands, and certainly the task is technically much more difficult before 24 weeks. The interval between each treatment is often judged arbitrarily, at least between the first and the second transfusions. We have found that the change in fetal haemoglobin concentration at this time can be difficult to predict and sometimes the packed cell volume falls suddenly (figure). Explanations include rapid sequestration of the transfused red cells by the fetal reticuloendothelial system, further haemolysis of the remaining fetal red cells, and leakage from the puncture site. Another possibility, which we are currently investigating, is that the fetoplacental vascular space is relatively contracted when the fetus is anaemic and its condition is poor. After transfusion fetal wellbeing improves and the vascular space expands, 
thereby diluting the red cells and lowering the haemoglobin concentration. This seems a likely explanation as the most rapid falls seem to occur among the sickest babies.

The volume of blood transfused must be sufficient to raise the packed cell volume to a reasonable level (usually about 0.4 ) without causing fluid overload, and normograms exist that provide a useful guide. The speed of transfusion is probably important, although the fetus seems to be able to cope with large volume transfusions sufficient at least to double the estimated fetoplacental volume. How such large transfusions are tolerated is unknown, but it may relate to the vast potential vascular space within the placenta which is capable of being further expanded by such vasodilating substances as atrial naturietic factor (and possibly prostacyclin) released in response to the fluid challenge.

The timing of further transfusions after the initial two is often easier to judge because the fall in packed cell volume against time becomes more consistent. The average drop of about $1-2 \% /$ day allows easy calculation of the timing of the next transfusion, but the traditional gap of two weeks (which was generated from the old intraperitoneal experience) seems often to be about right. The interval can, however, usually be increased to about three weeks after the third or fourth transfusion when the fetal condition has stabilised and only donor blood is circulating, and a combination of intravascular and intraperitoneal transfusions may extend this interval.

The route of transfusion needs to be chosen for each individual fetus, and probably to some extent depends on the stage of pregnancy. The cord root is used most frequently and is satisfactory so long as clear views are obtained. Though it is a relatively stable target it may be obscured by the fetus, which can usually be manoeuvred out of the way. As the pregnancy advances the fetus may not only overlie the cord root but also become less mobile making the intrahepatic portion of the umbilical vein sometimes preferable.

The timing of delivery has altered over the years, a balance being drawn between the chance of a vaginal delivery at a time when the

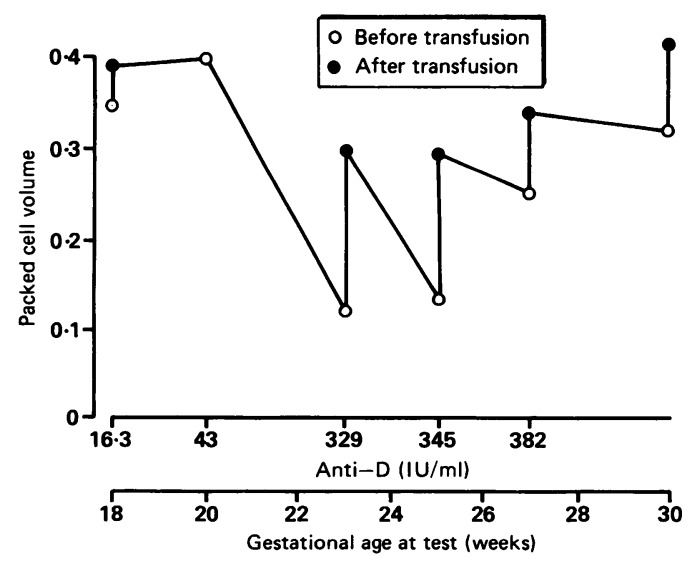

Typical response of packed cell volume to transfusion in a severely affected fetus. The packed cell volume in cord blood was unusually low. fetal lungs are likely to be mature against the additional risks of repeated intrauterine transfusion. Probably delivery timed for about 34 to 36 weeks is best, and certainly the continuation of the pregnancy beyond this time may be unnecessarily hazardous.

The outcome for these seriously ill fetuses when the intravascular route is used is surprisingly good, and about $80-90 \%$ of nonhydropic babies should survive compared with $70-80 \%$ of those with hydrops.

\section{Neonatal care and paediatric follow up}

The neonatal problems associated with severe rhesus disease virtually disappear when the fetus has received multiple transfusions, and because the baby's blood is virtually all donor in origin the haemoglobin concentration stays stable for a number of days. Exchange transfusion is rarely necessary, although a simple 'top up' may be required during the first few days of life. Of great importance is the fact that hyperbilirubinaemia does not usually occur, therefore reducing the need for an exchange transfusion. In contrast the hydropic baby even if born at full term may cause enormous paediatric problems that require intensive care, respiratory support, and-usually-multiple transfusions: mortality is often high.

Long term follow up of these severely affected babies is limited. Older published data would suggest that babies who have undergone intraperitoneal transfusion are no more likely than any other babies to develop mental or physical disabilities. The severely affected hydropic babies, however, usually died whereas nowadays, when intravascular techniques are used, they usually survive. Results of developmental testing of a group of previously severely affected babies from our own series showed no differences when compared with those of matched controls.

\section{Conclusions}

Rhesus isoimmune disease has become a rare condition. It is largely preventable by anti-D prophylaxis, although a small but appreciable group of cases remains. If untreated these babies have a high mortality or if they survive they provide major paediatric problems. Management in specialised centres where there are the facilities for carrying out intrauterine transfusions (often by the intravascular route) combined with high standards of neonatal care, has had a major impact on the outcome in these seriously ill babies.

Recommended reading

Gilmore DH, Whittle MJ. Modern management of rhesus disease. In: Chamberlain G, Drife J, eds. Contemporary reviews in obstetrics and gynaecology.
Butterworth-Heinemann, 1989:105-110.

Nicolaides KH, Rodeck CH, Mibashan RS, Kemp JR. Have Liley charts outlived their usefulness? Am $\mathcal{Y}$ Obstet Gynecol 1986;155:90-4.

Bovell PJ, Selinger M, Ferguson J, Giles J, MacKenzie IZ. Antenatal fetal blood sampling for the management of alloimmunized pregnancies: effect upon maternal anti-D immunized pregnancies: effect upon maternal ant-D

White CA, Goplerud CP, Kisker CT, Stehbens JA, Kitchell M, Taylor JC. Intrauterine fetal transfusion, 1965-1976, with an assessment of the surviving children. Am $\mathcal{J}$ Obstet Gynecol 1978;130:933-42. 